\title{
CYTOMEGALOVIRUS INFECTION AND CHRONIC IMMUNE-MEDIATED INFLAMMATORY DISEASES IN PEDIATRIC PATIENTS: CASES REPORTS AND RELEVANCE
}

Larissa Maria Oliveira Gonzaga ${ }^{1, \star}$, Ana Luisa Bagno de Almeida¹, Victória Carneiro Dal Moro', Samara Quadros Lobê, Anna Carolina Faria Moreira Gomes Tavares ${ }^{1}$, Flávia Patrícia Sena Teixeira Santos ${ }^{1}$

1.Universidade Federal de Minas Gerais, Belo Horizonte (MG), Brazil.

*Corresponding author: gonzagamlarissa@gmail.com

\section{BACKGROUND}

Human cytomegalovirus (HCMV) infection is recognized to be a precipitating factor of autoimmunity since it could be the trigger of many of these diseases. Besides that, HCMV infection can exacerbate some diseases manifestations, resulting in flares, or introduce itself similarly to them.

\section{CASE REPORT}

\section{Case 1}

Female, 12 years old, diagnosed with juvenile primary Sjögren's syndrome in 2017 treated initially with prednisone and methotrexate. She evolved with cutaneous vasculitis, arthritis, worsening of thrombocytopenia, lymphopenia, what motivated the switch to azathioprine. Then, with elevation of transaminases, myalgia and night fever, in-hospital investigation evidenced HCMV infection (positive IgG and IgM; HCMV pp65 antigen 50 cells), without evidence of invasive disease and ganciclovir $5 \mathrm{mg} / \mathrm{kg} / \mathrm{dose}$ was started. After seven days of treatment, she started with persistent fever and progressive pancytopenia, in addition to a drop in fibrinogen, elevation of triglycerides and transaminases, having been suspected of macrophage activation syndrome, which was confirmed by the finding of hemophagocytosis figures in the myelogram. In addition to ganciclovir, she was treated with methylprednisolone pulse therapy and cyclosporine, with good response.

\section{Case 2}

Female, 16 years old, diagnosed with juvenile systemic lupus erythematosus (only cutaneous, hematological and articular manifestations) in 2015, taking prednisone and cyclosporine, presenting elevated serum transaminase levels, intermittent fever and myalgia. In the investigation, an IgM serological turn was seen for cytomegalovirus and HCMV pp65 antigen was 13 cells. During the course of chronic CMV infection, she evolved with proteinuria (2,860 mg/day), retinal vasculitis, oligoarthritis and worsened cytopenia, which partially improved after treatment with ganciclovir. Afterwards she received cyclophosphamide and is now in complete remission with azathioprine.

\section{CONCLUSION}

Human cytomegalovirus infection in patients with autoimmune diseases is still challenging mainly because it mimics clinical manifestations and/or worsens underlying disease. Fever, cytopenia and liver dysfunction are the most common manifestations, but severe manifestations such as meningitis, pneumonitis and macrophage activation syndrome can occur. Diagnosis and treatment follow-up can be done by measuring HCMV pp65 antigenemia and/or HCMV PCR, the second being the gold standard technique for diagnosis, which is not always widely available. Leukopenia is a major limiter to the performance of antigenemia, very frequent in systemic lupus erythematosus. There are no well-defined recommendations for diagnosis and treatment of cytomegalovirus infection among patients with rheumatic diseases, which brings even more challenges for rheumatologist's clinical practice. 